Pak. J. Agri. Sci., Vol. 55(1), 63-71;2018

ISSN (Print) 0552-9034, ISSN (Online) 2076-0906

DOI: $10.21162 / P A K J A S / 18.5277$

http://www.pakjas.com.pk

\title{
PESTICIDES TOXICITY FOR Neoseiulus barkeri (ACARI: PHYTOSEIIDAE) AND NON-TARGET ORGANISMS
}

\author{
Muhammad Hamid Bashir ${ }^{1}$, Muhammad Zahid ${ }^{1, *}$, Muhammad Ahsan Khan', \\ Muhammad Shahid², Ahmad Kamran Khan' and Luqman Amrao \\ ${ }^{1}$ Department of Entomology, University of Agriculture, Faisalabad, Pakistan; ${ }^{2}$ Department of Biochemistry, \\ University of Agriculture, Faisalabad, Pakistan; Department of Plant Pathology, University of Agriculture, \\ Faisalabad, Pakistan. \\ *Corresponding author's e-mail: mhamzazahid@gmail.com
}

\begin{abstract}
Phytoseiidae have been used successfully as bio-control agents of sucking insect pests worldwide. Neoseiulus barkeri is potential predator of whiteflies, thrips and spider mites. To minimize toxic effects and develop IPM, integration of pesticides and predators in a manner which is safer for other organisms is key factor. The present study was planned for screening out pesticides being used against sucking pests i.e., buprofezin, spirotetramate, dimethoate, hexithiazox and imidacloprid for $N$. barkeri to find out compatibility. Leaf dip bioassay was conducted and pesticides were tested at five serial dilutions under laboratory conditions. Maximum mortality 17.5, 45, 82.5, 35 and 17.5 percent was observed after 144 hours exposure to pesticides at field relevant dose, respectively. Repellent effects of pesticides revealed that escape from arena was highest in the start and then gradually decreased. Lowest hemolytic activity (78.56\%) for imidacloprid and highest (97.76\%) for dimethoate, and Staphylococcus aureus biofilm inhibition $43 \%$ for buprofezin and $23 \%$ for dimethoate was observed. $\mathrm{LC}_{50}$ of imidacloprid (26526) and buprofezin (7209) declared it safer while spirotetramate and hexithiazox were moderately harmful to N. barkeri. Dimethoate was highly hazardous for $N$. barkeri due to highest mortality and lowest $\mathrm{LT}_{50}$ (27.75), hence is not recommended in IPM module.
\end{abstract}

Keywords: Neoseiulus barkeri, reduced-risk pesticides, mortality, biofilm inhibition, pest management.

\section{INTRODUCTION}

Protection of crops from insect pests is the main focusing approach for maximizing existing crop yields and economic returns. The use of pesticides has become an integral part of our pest management approach (Lechenet et al., 2014). These chemicals are giving satisfactory results but in parallel they also impose serious threats like environmental pollution, risk to beneficial fauna and human health. Due to injudicious use of pesticides, there are many reports for the development of pesticides resistance in insect pests (Abang et al., 2013; Attia et al., 2015). This adverse situation directs the agricultural experts to rely on human and ecosystem friendly alternate approach. Biological control is considered as the most promising for the conservation of natural fauna and reduction of toxic residues in our ecosystem (Ajinath et al., 2013). The predatory mites are being commercially used in various parts of the world and are considered as potential predators in biological systems (Chant and McMurtry, 2007; Mallik et al., 2010; Szabo et al., 2014). In recent years, the use of chemical and biological control in an integrated manner has become a popular IPM approach. The main objective of such tactic is to use lower doses of pesticides or apply safer pesticides along with predators, to lessen the pesticides burden on ecosystems, decrease pesticide resistance in pests, and to increase existing crop yields and ensure good human health (Hosny et al., 2009).

In Pakistan major threat of sucking insect-pests, particularly whitefly and spider mites, has become very crucial (Rafiq et al., 2008; Ahmad et al., 2010; Mamoon-ur-Rashid, 2011) and for their control huge investments are being spent on pesticides (Malik, 2014). The predatory mite, Neoseiulus barkeri Hughes (Acari: Phytoseiidae) is a generalist predator (McMurtry et al., 2013). Number of studies revealed the predatory potential of $N$. barkeri against wide range of pests including Thrips tabaci (Hansen, 1988; Bakker and Sabelis, 1989; Bonde, 1989; Jafari et al., 2013), broad mite, Polyphagotarsonemus latus (Fan and Petitt, 1994), bulb scale mites, Steneotarsonemus laticeps (Messelink and HolsteinSaj, 2007; Messelink, 2012), date mite, Oligonychus afrasiaticus (Negm et al., 2014) and Aleuroglyphus ovatus (Xia et al., 2012). Keeping in view, the predatory potential of this predatory mite, the focus is to use selective pesticides along with it which are least toxic for this predator. Safer and compatible pesticides are the main pillars of biological and chemical control programs in IPM approaches. Now a day, it became evident that the newer pesticides fit the reduced-risk profile, but these may not be necessarily safer for predatory mites (Villanueva and Walgenbach, 2005; Bostanian et al., 2009; Lefebvre et al., 2012). The varying level of toxicity 
ranging from harmless to harmful effects of pesticides against predatory mites has been reported (Jansen, 2010). Imidacloprid, fenbutain oxide, acetamiprid, buprofezin, fenobucarb, dinotefuran, validamycin, carbendazim, hexithiazox and sulfur were safer against different predatory mites (Castagnoli et al., 2005; Kongchuensin and Takafuji, 2006; Sanatgar et al., 2011; Fiedler and Sosnowska, 2014). Pesticides such as etofenprox, spinosad, chloropyriphos and dimethoate had harmful effects (Bostanian and Akalach, 2006; Alzoubi and Cobanoglu, 2008), while thiamethoxam was low to moderately harmful for predatory mites (Tirello et al., 2013). Emmamectin benzoate was reported to be highly toxic, while indoxacarb was a safer pesticide against tested predatory mite (Bernard et al., 2010). Different pesticides have proved to be toxic for human beings and other non-target organisms and reported to affect human health adversely (Son et al., 2010; Alvanja and Bonner, 2012). Imidacloprid, thiamethoxam, clothianidin and acetamiprid were found highly toxic to beneficial fauna in comparison with spirotetramate, buprofezin and fipronil (Kumar et al., 2012). Dimethoate had significantly decreased the body size, haemocyte counts and morphometric factors in carabid beetle (Giglio et al., 2011). Sulfonylurea herbicides-chlorsulfuron, metasulfuron methyl and thifensulfuron methyl reduced the growth rate of fluorescent bacteria Pseudomonas strains (Boldt and Jacobson, 1998). Methyl isothiocyanate caused an increase in gram positive bacteria and decrease in gram negative bacteria (Ibekwe et al., 2001).

In Pakistan, no work has been carried out to screen the safer pesticides for predators and to study the impact of pesticides on human and some non-target organisms present in nature.

\section{MATERIALS AND METHODS}

Source of mites: The native predatory mite $N$. barkeri was collected from cotton fields of University of Agriculture, Faisalabad, Pakistan and reared in the laboratory since 2010, having no exposure to pesticides used for experimentation. Stock culture was reared on stored grain mite Rhizoglyphus tritici in growth chamber at $26 \pm 2{ }^{\circ} \mathrm{C}$ temperature, $65 \pm 5 \%$ relative humidity and 12:12 (L:D) photoperiod. The culture was kept in small petri dishes of $5.5 \mathrm{~cm}$ diameter placed on foam of $12 \mathrm{~cm}$ diameter which was soaked in water in large petri dishes (14 cm diameter).

Table 1. Pesticides along with trade names, groups, concentration (ppm) and field recommended dose rates.

\begin{tabular}{lllcc}
\hline $\begin{array}{l}\text { Name of } \\
\text { pesticide }\end{array}$ & Trade name & Group & $\begin{array}{c}\text { Concentration in } \\
\text { sprayable material (ppm) }\end{array}$ & $\begin{array}{c}\text { Recommended } \\
\text { dose/acre/100 L water }\end{array}$ \\
\hline buprofezin & Starter 25\% WP & Insect Growth Regulator & 1250 & $500 \mathrm{GM}$ \\
spirotetramate & Movinto & Keto-enolen & 720 & $150 \mathrm{ML}$ \\
dimethoate & Sanitox 40 EC & Organophosphate & 1600 & $400 \mathrm{ML}$ \\
hexithiazox & Nissuron & Thiazolidine and Carboxamide & 125 & $125 \mathrm{GM}$ \\
imidacloprid & Confidor 20\% SC & Neonicotinoid & 500 & $250 \mathrm{ML}$ \\
\hline
\end{tabular}

Pesticides: The pesticides were selected from diverse groups which are being commonly used against sucking insect pests (Table 1). These were purchased from the local market. Serial dilutions were prepared in acetone starting from the field relevant dose.

Toxicity to adults: Leaf discs of $1.7 \mathrm{~cm}$ diameter were prepared from three months old brinjal, Solanum melongena (Solanaceae) leaves with the help of cork borer. Arenas were prepared by keeping foam (12cm diameter) in $14 \mathrm{~cm}$ diameter petri dish containing water as barrier to prevent escape of predatory mites. The leaf discs were dipped in different concentrations of pesticides for 10 seconds. These discs were allowed to dry for 30 minutes at room temperature (Kongchuensin and Takafuji, 2006). Predatory mites were fed with respective diet before experimentation to ensure mortality occurred due to pesticide not due to starvation. Ten newly developed females were placed on each leaf disc. Immatures of $R$. tritici were offered as food source daily. These mites were added in the arenas to replace the consumed preys to keep the predator prey ratio (1:3) constant. Moreover, absconded predatory mites were excluded from data. Those predatory mites were considered dead which showed no response when touched with a fine needle. Mortality and escape data was recorded after every 24 hours till 144 hours.

Statistical analysis: Data were analyzed statistically by calculating means, standard errors, percentages and two way analysis of variance (ANOVA) and comparison of means were separated by least significant difference (LSD) $(\mathrm{P}<0.05)$. $\mathrm{LC}_{50}$ and $\mathrm{LT}_{50}$ values for all tested pesticides were calculated with probit analysis by using statistical software Minitab17. Toxicity categories of tested pesticides were evaluated according to IOBC (International Organization for Biological and Integrated Control) criteria (Jansen, 2010).

\section{RESULTS}

Toxicity to adult female Neoseiulus barkeri: Pesticides at different concentrations and time intervals were evaluated against $N$. barkeri, and different effects were observed. Highly significant differences in mortality at different concentrations $(\mathrm{F}=9.01,119.03,268.29,76.16,20.33$, df $=$ $5,108, \mathrm{P} \leq 0.000)$ and time intervals $(\mathrm{F}=11.07,9.49,31.05$, 7.33, 23.02, df $=5,108, \mathrm{P} \leq 0.000)$ were observed for buprofezin, spirotetramate, dimethoate, hexithiazox and imidacloprid respectively. Escape of $N$. barkeri from leaf 
arena depends upon toxicity of tested pesticides, it was highest in the start of experiment but gradually decreased with the passage of time due to acclimatization. It was significantly different at different concentrations $(\mathrm{F}=12.65, \mathrm{df}=5,108 \mathrm{P} \leq$ $0.000)$ and time intervals $(\mathrm{F}=3.74, \mathrm{df}=5,108 \mathrm{P}=0.004)$ for buprofezin (Table 2b). It was not different at different time intervals $(\mathrm{F}=1.76,1.64$, df $=5,108 \mathrm{P}=0.127,0.156)$ and significantly different at different concentrations $(\mathrm{F}=10.24$, 8.42 , df $=5,108 \mathrm{P} \leq 0.000$ ) for spirotetramate and dimethoate respectively (Table $3 \mathrm{~b}, 4 \mathrm{~b}$ ). It was significantly different at

Table 2a. Mortality (\%) of $N$. barkeri $(n=10)$ observed on leaf arenas at different time intervals for buprofezin $($ Mean \pm SE).

\begin{tabular}{|c|c|c|c|c|c|c|c|}
\hline \multirow{2}{*}{$\begin{array}{l}\text { Time } \\
\text { (hours) }\end{array}$} & \multicolumn{6}{|c|}{ Dose (ppm) } & \multirow[t]{2}{*}{ Mean } \\
\hline & 1250 & 625 & 312.50 & 156.25 & 78.12 & Control & \\
\hline 24 & $2.50 \pm 2.50$ & $2.50 \pm 2.50$ & $0.00 \pm 0.00$ & $0.00 \pm 0.00$ & $0.00 \pm 0.00$ & $0.00 \pm 0.00$ & $0.83 \pm 0.58 \mathrm{E}$ \\
\hline 48 & $5.00 \pm 2.89$ & $5.00 \pm 2.89$ & $2.50 \pm 2.50$ & $2.50 \pm 2.50$ & $0.00 \pm 0.00$ & $2.50 \pm 2.50$ & $2.92 \pm 0.95 \mathrm{DE}$ \\
\hline 72 & $5.00 \pm 2.89$ & $5.00 \pm 2.89$ & $2.50 \pm 2.50$ & $2.50 \pm 2.50$ & $2.50 \pm 2.50$ & $5.00 \pm 2.89$ & $3.75 \pm 1.01 \mathrm{CD}$ \\
\hline 96 & $12.50 \pm 2.50$ & $7.50 \pm 2.50$ & $5.00 \pm 2.89$ & $2.50 \pm 2.50$ & $2.50 \pm 2.50$ & $5.00 \pm 2.89$ & $5.83 \pm 1.19 \mathrm{BC}$ \\
\hline 120 & $15.00 \pm 2.89$ & $12.50 \pm 2.50$ & $10.00 \pm 0.00$ & $2.50 \pm 2.50$ & $5.00 \pm 2.89$ & $5.00 \pm 2.89$ & $8.33 \pm 1.30 \mathrm{AB}$ \\
\hline 144 & $17.50 \pm 2.50$ & $12.50 \pm 2.50$ & $10.00 \pm 0.00$ & $2.50 \pm 2.50$ & $5.00 \pm 2.89$ & $7.50 \pm 2.50$ & $9.17 \pm 1.33 \mathrm{~A}$ \\
\hline Total & $9.58 \pm 1.53 \mathrm{~A}$ & $7.50 \pm 1.24 \mathrm{AB}$ & $5.00 \pm 1.04 \mathrm{BC}$ & $2.08 \pm 0.85 \mathrm{D}$ & $2.50 \pm 0.90 \mathrm{CD}$ & $4.17 \pm 1.03 \mathrm{CD}$ & \\
\hline
\end{tabular}

Means with the same letter in a row or in a column are not significantly different by Fisher's LSD (P $>0.05)$; T1 $=$ Field relevant dose $(1250 \mathrm{ppm}), \mathrm{T} 2=1 / 2$ of field relevant dose $(625 \mathrm{ppm}), \mathrm{T} 3=1 / 4$ of field relevant dose $(312.50 \mathrm{ppm}), \mathrm{T} 4=1 / 8$ of field relevant dose $(156.25 \mathrm{ppm}), \mathrm{T} 5=1 / 16$ of field relevant dose $(78.12 \mathrm{ppm})$ and $\mathrm{T} 6=$ Control (Acetone)

Table 2b. Rate of escape (\%) of $N$. barkeri $(\mathrm{n}=10)$ at different time intervals for buprofezin $(\mathrm{Mean} \pm \mathrm{SE})$.

\begin{tabular}{|c|c|c|c|c|c|c|c|}
\hline \multirow{2}{*}{$\begin{array}{l}\text { Time } \\
\text { (hours) }\end{array}$} & \multicolumn{6}{|c|}{ Dose (ppm) } & \multirow[t]{2}{*}{ Mean } \\
\hline & 1250 & 625 & 312.50 & 156.25 & 78.12 & Control & \\
\hline 24 & $10.00 \pm 0.00$ & $10.00 \pm 0.00$ & $12.50 \pm 2.50$ & $7.50 \pm 2.50$ & $7.50 \pm 2.50$ & $5.00 \pm 2.89$ & $8.75 \pm 0.92 \mathrm{C}$ \\
\hline 48 & $12.50 \pm 2.50$ & $12.50 \pm 2.50$ & $12.50 \pm 2.50$ & $10.00 \pm 0.00$ & $10.00 \pm 0.00$ & $5.00 \pm 2.89$ & $10.42 \pm 0.95 \mathrm{BC}$ \\
\hline 72 & $12.50 \pm 2.50$ & $15.00 \pm 2.89$ & $12.50 \pm 2.50$ & $10.00 \pm 0.00$ & $10.00 \pm 0.00$ & $7.50 \pm 2.50$ & $11.25 \pm 0.92 \mathrm{ABC}$ \\
\hline 96 & $15.00 \pm 2.89$ & $17.50 \pm 2.50$ & $12.50 \pm 2.50$ & $10.00 \pm 0.00$ & $10.00 \pm 0.00$ & $7.50 \pm 2.50$ & $12.08 \pm 1.04 \mathrm{AB}$ \\
\hline 120 & $17.50 \pm 2.50$ & $17.50 \pm 2.50$ & $15.00 \pm 2.89$ & $12.50 \pm 2.50$ & $10.00 \pm 0.00$ & $7.50 \pm 2.50$ & $13.33 \pm 1.15 \mathrm{~A}$ \\
\hline 144 & $17.50 \pm 2.50$ & $17.50 \pm 2.50$ & $15.00 \pm 2.89$ & $12.50 \pm 2.50$ & $12.50 \pm 2.50$ & $5.00 \pm 2.89$ & $13.33 \pm 1.30 \mathrm{~A}$ \\
\hline Total & $14.17 \pm 1.03 \mathrm{~A}$ & $15.00 \pm 1.04 \mathrm{~A}$ & $13.33 \pm 0.98 \mathrm{~A}$ & $10.42 \pm 0.73 B$ & $10.00 \pm 0.60 \mathrm{~B}$ & $6.25 \pm 1.01 \mathrm{C}$ & \\
\hline
\end{tabular}

Means with the same letter in a row or in a column are not significantly different by Fisher's LSD $(\mathrm{P}>0.05)$; T1 $=$ Field relevant dose $(1250 \mathrm{ppm}), \mathrm{T} 2=1 / 2$ of field relevant dose $(625 \mathrm{ppm}), \mathrm{T} 3=1 / 4$ of field relevant dose $(312.50 \mathrm{ppm}), \mathrm{T} 4=1 / 8$ of field relevant dose $(156.25 \mathrm{ppm}), \mathrm{T} 5=1 / 16$ of field relevant dose $(78.12 \mathrm{ppm})$ and $\mathrm{T} 6=\mathrm{Control}$ (Acetone)

Table 3a. Mortality $(\%)$ of $N$. barkeri $(n=10)$ observed on leaf arenas at different time intervals for spirotetramate (Mean+SE).

\begin{tabular}{|c|c|c|c|c|c|c|c|}
\hline \multirow{2}{*}{$\begin{array}{l}\text { Time } \\
\text { (hours) }\end{array}$} & \multicolumn{6}{|c|}{ Dose (ppm) } & \multirow[t]{2}{*}{ Mean } \\
\hline & 720 & 360 & 180 & 90 & 45 & Control & \\
\hline 24 & $22.50 \pm 2.50$ & $15.00 \pm 2.89$ & $12.50 \pm 2.50$ & $7.50 \pm 2.50$ & $5.00 \pm 2.89$ & $0.00 \pm 0.00$ & $10.42 \pm 1.75 \mathrm{C}$ \\
\hline 48 & $32.50 \pm 4.79$ & $25.00 \pm 2.89$ & $15.00 \pm 2.89$ & $10.00 \pm 0.00$ & $7.50 \pm 2.50$ & $0.00 \pm 0.00$ & $15.00 \pm 2.48 B$ \\
\hline 72 & $37.50 \pm 4.79$ & $30.00 \pm 4.08$ & $15.00 \pm 2.89$ & $10.00 \pm 0.00$ & $7.50 \pm 2.50$ & $0.00 \pm 0.00$ & $16.67 \pm 2.93 \mathrm{AB}$ \\
\hline 96 & $42.50 \pm 4.79$ & $30.00 \pm 4.08$ & $17.50 \pm 2.50$ & $12.50 \pm 2.50$ & $7.50 \pm 2.50$ & $5.00 \pm 2.89$ & $19.17 \pm 3.00 \mathrm{~A}$ \\
\hline 120 & $45.00 \pm 2.89$ & $32.50 \pm 4.79$ & $17.50 \pm 2.50$ & $12.50 \pm 2.50$ & $7.50 \pm 2.50$ & $5.00 \pm 2.89$ & $20.00 \pm 3.19 \mathrm{~A}$ \\
\hline 144 & $45.00 \pm 2.89$ & $32.50 \pm 4.79$ & $17.50 \pm 2.50$ & $12.50 \pm 2.50$ & $7.50 \pm 2.50$ & $5.00 \pm 2.89$ & $20.00 \pm 3.19 \mathrm{~A}$ \\
\hline Total & $37.50 \pm 2.19 \mathrm{~A}$ & $27.50 \pm 1.93 \mathrm{~B}$ & $15.83 \pm 1.03 \mathrm{C}$ & $10.83 \pm 0.83 \mathrm{D}$ & $7.08 \pm 0.95 \mathrm{E}$ & $2.50 \pm 0.90 \mathrm{~F}$ & \\
\hline
\end{tabular}

Means with the same letter in a row or in a column are not significantly different by Fisher's LSD (P>0.05).

Table 3b. Rate of escape $(\%)$ of $N$. barkeri $(n=10)$ at different time intervals for spirotetramate $(\mathrm{Mean} \pm \mathrm{SE})$.

\begin{tabular}{|c|c|c|c|c|c|c|c|}
\hline \multirow{2}{*}{$\begin{array}{l}\text { Time } \\
\text { (hours) }\end{array}$} & \multicolumn{6}{|c|}{ Dose (ppm) } & \multirow[t]{2}{*}{ Mean } \\
\hline & 720 & 360 & 180 & 90 & 45 & Control & \\
\hline 24 & $10.00 \pm 0.00$ & $10.00 \pm 0.00$ & $10.00 \pm 0.00$ & $12.50 \pm 2.50$ & $10.00 \pm 0.00$ & $5.00 \pm 2.89$ & $9.58 \pm 0.73 \mathrm{~A}$ \\
\hline 48 & $10.00 \pm 0.00$ & $12.50 \pm 2.50$ & $10.00 \pm 0.00$ & $12.50 \pm 2.50$ & $10.00 \pm 0.00$ & $5.00 \pm 2.89$ & $10.00 \pm 0.85 \mathrm{~A}$ \\
\hline 72 & $12.50 \pm 2.50$ & $12.50 \pm 2.50$ & $12.50 \pm 2.50$ & $15.00 \pm 2.89$ & $10.00 \pm 0.00$ & $5.00 \pm 2.89$ & $11.25 \pm 1.10 \mathrm{~A}$ \\
\hline 96 & $12.50 \pm 2.50$ & $12.50 \pm 2.50$ & $12.50 \pm 2.50$ & $15.00 \pm 2.89$ & $12.50 \pm 2.50$ & $5.00 \pm 2.89$ & $11.67 \pm 1.15 \mathrm{~A}$ \\
\hline 120 & $12.50 \pm 2.50$ & $12.50 \pm 2.50$ & $12.50 \pm 2.50$ & $17.50 \pm 2.50$ & $12.50 \pm 2.50$ & $7.50 \pm 2.50$ & $12.50 \pm 1.09 \mathrm{~A}$ \\
\hline 144 & $12.50 \pm 2.50$ & $12.50 \pm 2.50$ & $12.50 \pm 2.50$ & $17.50 \pm 2.50$ & $12.50 \pm 2.50$ & $7.50 \pm 2.50$ & $12.50 \pm 1.09 \mathrm{~A}$ \\
\hline Total & $11.67 \pm 0.78 \mathrm{~B}$ & $12.08 \pm 0.85 \mathrm{~B}$ & $11.67 \pm 0.78 \mathrm{~B}$ & $15.00 \pm 1.04 \mathrm{~A}$ & $11.25 \pm 0.69 \mathrm{~B}$ & $5.83 \pm 1.03 \mathrm{C}$ & \\
\hline
\end{tabular}


Bashir, Zahid, Khan, Shahid, Khan \& Amrao

Table 4a. Mmortality (\%) of $N$. barkeri $(n=10)$ observed on leaf arenas at different time intervals for dimethoate $(\mathrm{Mean} \pm \mathrm{SE})$.

\begin{tabular}{|c|c|c|c|c|c|c|c|}
\hline \multirow{2}{*}{$\begin{array}{l}\text { Time } \\
\text { (hours) }\end{array}$} & \multicolumn{6}{|c|}{ Dose (ppm) } & \multirow[t]{2}{*}{ Mean } \\
\hline & 1600 & 800 & 400 & 200 & 100 & Control & \\
\hline 24 & $37.50 \pm 2.50 \mathrm{f}$ & $25.00 \pm 2.89 \mathrm{ghi}$ & $15.00 \pm 2.89 \mathrm{jkl}$ & $7.50 \pm 2.501 \mathrm{mn}$ & $5.00 \pm 2.89 \mathrm{mn}$ & $0.00 \pm 0.00 n$ & $15.00 \pm 2.82 \mathrm{E}$ \\
\hline 48 & $65.00 \pm 2.89 b c$ & $50.00 \pm 4.08 \mathrm{e}$ & $27.50 \pm 6.29 \mathrm{gh}$ & $17.50 \pm 2.50 \mathrm{ijk}$ & $5.00 \pm 2.89 \mathrm{mn}$ & $2.50 \pm 2.50 \mathrm{mn}$ & $27.92 \pm 4.96 \mathrm{C}$ \\
\hline 72 & $70.00 \pm 4.08 b$ & $52.50 \pm 2.50 \mathrm{de}$ & $27.50 \pm 6.29 \mathrm{gh}$ & $17.50 \pm 2.50 \mathrm{ijk}$ & $7.50 \pm 2.501 \mathrm{mn}$ & $5.00 \pm 2.89 \mathrm{mn}$ & $30.00 \pm 5.14 \mathrm{BC}$ \\
\hline 96 & $72.50 \pm 2.50 b$ & $55.00 \pm 2.89 \mathrm{de}$ & $30.00 \pm 4.08 \mathrm{fg}$ & $20.00 \pm 4.08 \mathrm{hij}$ & $10.00 \pm 0.00 \mathrm{klm}$ & $5.00 \pm 2.89 \mathrm{mn}$ & $32.08 \pm 5.18 \mathrm{AB}$ \\
\hline 120 & $82.50 \pm 2.50 \mathrm{a}$ & $60.00 \pm 4.08 \mathrm{~cd}$ & $30.00 \pm 4.08 \mathrm{fg}$ & $20.00 \pm 4.08 \mathrm{hij}$ & $10.00 \pm 0.00 \mathrm{klm}$ & $7.50 \pm 2.501 \mathrm{mn}$ & $35.00 \pm 5.84 \mathrm{~A}$ \\
\hline 144 & $82.50 \pm 2.50 \mathrm{a}$ & $65.00 \pm 2.89 b c$ & $30.00 \pm 4.08 \mathrm{fg}$ & $20.00 \pm 4.08 \mathrm{hij}$ & $10.00 \pm 0.00 \mathrm{klm}$ & $7.50 \pm 2.501 \mathrm{mn}$ & $35.83 \pm 4.58 \mathrm{D}$ \\
\hline Total & $68.33 \pm 5.93 \mathrm{~A}$ & $51.25 \pm 2.91 \mathrm{~A}$ & $26.67 \pm 2.06 \mathrm{~B}$ & $17.08 \pm 1.53 \mathrm{C}$ & $7.92 \pm 0.85 \mathrm{D}$ & $4.58 \pm 1.04 \mathrm{D}$ & \\
\hline
\end{tabular}

Means with the same letter in a row or in a column are not significantly different by Fisher's LSD ( $\mathrm{P}>0.05)$.

Table 4b. Rate of escape (\%) of $N$. barkeri $(\mathrm{n}=10)$ at different time intervals for dimethoate (Mean $\pm \mathrm{SE})$.

\begin{tabular}{|c|c|c|c|c|c|c|c|}
\hline \multirow{2}{*}{$\begin{array}{l}\text { Time } \\
\text { (hours) }\end{array}$} & \multicolumn{6}{|c|}{ Dose (ppm) } & \multirow[t]{2}{*}{ Mean } \\
\hline & 1600 & 800 & 400 & 200 & 100 & Control & \\
\hline 24 & $15.00 \pm 2.89 \mathrm{ab}$ & $10.00 \pm 0.00 \mathrm{bcd}$ & $10.00 \pm 0.00 \mathrm{bcd}$ & $10.00 \pm 0.00 \mathrm{bcd}$ & $12.50 \pm 2.50 \mathrm{abc}$ & $5.00 \pm 2.89 \mathrm{de}$ & $10.42 \pm 0.95 \mathrm{~A}$ \\
\hline 48 & $15.00 \pm 2.89 \mathrm{ab}$ & $12.50 \pm 2.50 \mathrm{abc}$ & $10.00 \pm 0.00 \mathrm{bcd}$ & $10.00 \pm 0.00 \mathrm{bcd}$ & $12.50 \pm 2.50 \mathrm{abc}$ & $5.00 \pm 2.89 \mathrm{de}$ & $10.83 \pm 1.03 \mathrm{~A}$ \\
\hline 72 & $17.50 \pm 2.50 \mathrm{a}$ & $15.00 \pm 2.89 \mathrm{ab}$ & $12.50 \pm 2.50 \mathrm{abc}$ & $12.50 \pm 2.50 \mathrm{abc}$ & $12.50 \pm 2.50 \mathrm{abc}$ & $5.00 \pm 2.89 \mathrm{de}$ & $12.50 \pm 1.24 \mathrm{~A}$ \\
\hline 96 & $17.50 \pm 2.50 \mathrm{a}$ & $15.00 \pm 2.89 \mathrm{ab}$ & $12.50 \pm 2.50 \mathrm{abc}$ & $12.50 \pm 2.50 \mathrm{abc}$ & $15.00 \pm 2.89 \mathrm{ab}$ & $7.50 \pm 2.50 \mathrm{~cd}$ & $13.33 \pm 1.15 \mathrm{~A}$ \\
\hline 120 & $17.50 \pm 2.50 \mathrm{a}$ & $15.00 \pm 2.89 \mathrm{ab}$ & $12.50 \pm 2.50 \mathrm{abc}$ & $12.50 \pm 2.50 \mathrm{abc}$ & $15.00 \pm 2.89 \mathrm{ab}$ & $7.50 \pm 2.50 \mathrm{~cd}$ & $13.33 \pm 1.15 \mathrm{~A}$ \\
\hline 144 & $17.50 \pm 2.50 \mathrm{a}$ & $15.00 \pm 2.89 \mathrm{ab}$ & $15.00 \pm 2.89 \mathrm{ab}$ & $15.00 \pm 2.89 \mathrm{ab}$ & $15.00 \pm 2.89 \mathrm{ab}$ & $7.50 \pm 2.50 \mathrm{~cd}$ & $14.17 \pm 1.51 \mathrm{~A}$ \\
\hline Total & $16.67 \pm 1.57 \mathrm{~B}$ & $13.75 \pm 1.01 \mathrm{AB}$ & $12.08 \pm 0.85 \mathrm{AB}$ & $12.08 \pm 0.85 \mathrm{~A}$ & $13.75 \pm 1.01 \mathrm{~A}$ & $6.25 \pm 1.01 \mathrm{AB}$ & \\
\hline
\end{tabular}

Table 5a. Mortality (\%) of $N$. barkeri (n=10) observed on leaf arenas at different time intervals for hexithiazox $($ Mean \pm SE).

\begin{tabular}{|c|c|c|c|c|c|c|c|}
\hline \multirow{2}{*}{$\begin{array}{l}\text { Time } \\
\text { (hours) }\end{array}$} & \multicolumn{6}{|c|}{ Dose (ppm) } & \multirow[t]{2}{*}{ Mean } \\
\hline & 125 & 62.50 & 31.25 & 15.62 & 7.81 & Control & \\
\hline 24 & $15.00 \pm 2.89$ & $10.00 \pm 0.00$ & $5.00 \pm 2.89$ & $2.50 \pm 2.50$ & $2.50 \pm 2.50$ & $0.00 \pm 0.00$ & $5.83 \pm 1.33 \mathrm{C}$ \\
\hline 48 & $25.00 \pm 2.89$ & $15.00 \pm 2.89$ & $10.00 \pm 0.00$ & $5.00 \pm 2.89$ & $2.50 \pm 2.50$ & $2.50 \pm 2.50$ & $10.00 \pm 1.90 \mathrm{~B}$ \\
\hline 72 & $27.50 \pm 2.50$ & $17.50 \pm 4.79$ & $10.00 \pm 0.00$ & $7.50 \pm 2.50$ & $2.50 \pm 2.50$ & $2.50 \pm 2.50$ & $11.25 \pm 2.11 \mathrm{AB}$ \\
\hline 96 & $30.00 \pm 4.08$ & $20.00 \pm 4.08$ & $10.00 \pm 0.00$ & $7.50 \pm 2.50$ & $2.50 \pm 2.50$ & $5.00 \pm 2.89$ & $12.50 \pm 2.27 \mathrm{AB}$ \\
\hline 120 & $35.00 \pm 2.89$ & $20.00 \pm 4.08$ & $10.00 \pm 0.00$ & $7.50 \pm 2.50$ & $5.00 \pm 2.89$ & $5.00 \pm 2.89$ & $13.75 \pm 2.47 \mathrm{~A}$ \\
\hline 144 & $35.00 \pm 2.89$ & $20.00 \pm 4.08$ & $10.00 \pm 0.00$ & $7.50 \pm 2.50$ & $5.00 \pm 2.89$ & $5.00 \pm 2.89$ & $13.75 \pm 2.47 \mathrm{~A}$ \\
\hline Total & $27.92 \pm 1.80 \mathrm{~A}$ & $17.08 \pm 1.53 \mathrm{~B}$ & $9.17 \pm 0.58 \mathrm{C}$ & $6.25 \pm 1.01 \mathrm{CD}$ & $3.33 \pm 0.98 \mathrm{D}$ & $3.33 \pm 0.98 \mathrm{D}$ & \\
\hline
\end{tabular}

Means with the same letter in a row or in a column are not significantly different by Fisher's LSD ( $\mathrm{P}>0.05)$.

Table 5b. Rate of escape (\%) of $N$. barkeri $(\mathrm{n}=10)$ at different time intervals for hexithiazox (Mean $\pm \mathrm{SE})$.

\begin{tabular}{|c|c|c|c|c|c|c|c|}
\hline \multirow{2}{*}{$\begin{array}{l}\text { Time } \\
\text { (hours) }\end{array}$} & \multicolumn{6}{|c|}{ Dose (ppm) } & \multirow[t]{2}{*}{ Mean } \\
\hline & 125 & 62.50 & 31.25 & 15.62 & 7.81 & Control & \\
\hline 24 & $10.00 \pm 0.00$ & $10.00 \pm 0.00$ & $7.50 \pm 2.50$ & $7.50 \pm 2.50$ & $7.50 \pm 2.50$ & $5.00 \pm 2.89$ & $7.92 \pm 0.85 B$ \\
\hline 48 & $10.00 \pm 0.00$ & $10.00 \pm 0.00$ & $7.50 \pm 2.50$ & $7.50 \pm 2.50$ & $7.50 \pm 2.50$ & $5.00 \pm 2.89$ & $7.92 \pm 0.85 \mathrm{~B}$ \\
\hline 72 & $12.50 \pm 2.50$ & $12.50 \pm 2.50$ & $10.00 \pm 0.00$ & $7.50 \pm 2.50$ & $7.50 \pm 2.50$ & $5.00 \pm 2.89$ & $9.17 \pm 1.03 \mathrm{AB}$ \\
\hline 96 & $15.00 \pm 2.89$ & $15.00 \pm 2.89$ & $10.00 \pm 0.00$ & $10.00 \pm 0.00$ & $7.50 \pm 2.50$ & $5.00 \pm 2.89$ & $10.42 \pm 1.12 \mathrm{AB}$ \\
\hline 120 & $15.00 \pm 2.89$ & $15.00 \pm 2.89$ & $12.50 \pm 2.50$ & $10.00 \pm 0.00$ & $10.00 \pm 0.00$ & $7.50 \pm 2.50$ & $11.67 \pm 0.98 \mathrm{~A}$ \\
\hline 144 & $15.00 \pm 2.89$ & $15.00 \pm 2.89$ & $12.50 \pm 2.50$ & $10.00 \pm 0.00$ & $10.00 \pm 0.00$ & $7.50 \pm 2.50$ & $11.67 \pm 0.98 \mathrm{~A}$ \\
\hline Total & $12.92 \pm 0.95 \mathrm{~A}$ & $12.92 \pm 0.95 \mathrm{~A}$ & $10.00 \pm 0.85 \mathrm{~B}$ & $8.75 \pm 0.69 \mathrm{~B}$ & $8.33 \pm 0.78 \mathrm{BC}$ & $5.83 \pm 1.03 \mathrm{C}$ & \\
\hline
\end{tabular}

Means with the same letter in a row or in a column are not significantly different by Fisher's LSD ( $>>0.05)$.

different time intervals $(\mathrm{F}=3.62,2.40 \mathrm{df}=5,108, \mathrm{P}=0.005$ $0.042)$ and concentrations $(\mathrm{F}=9.38,7.69, \mathrm{df}=5,108, \mathrm{P} \leq 0.000)$ for hexithiazox and imidacloprid respectively (Table $5 \mathrm{~b}, 6 \mathrm{~b})$. Buprofezin was harmless for $N$. barkeri, maximum mortality $(17.50 \%)$ was observed at field-relevant dose (1250 ppm) after 144 hours, while minimum mortality (2.50\%) at same concentration and at 625 ppm after 24 hours (Table 2a). There was non-significant interaction of time and concentrations for mites escape $(\mathrm{F}=0.38, \mathrm{df}=25,108, \mathrm{P}=0.996)$ and mortality $(\mathrm{F}=0.82, \mathrm{df}=25,108, \mathrm{P}=0.699)$ were observed (Table $2 \mathrm{a}, \mathrm{b})$. Spirotetramate caused maximum mortality $(45 \%)$ at field relevant dose $(720 \mathrm{ppm})$ after 120 hours and minimum mortality $(5.00 \%)$ at 45 ppm after 24 hours (Table 3a). There was non-significant interaction of time and spirotetramate 
Table 6a. Mortality (\%) of $N$. barkeri $(\mathrm{n}=10)$ observed on leaf arenas at different time intervals for imidacloprid $($ Mean \pm SE).

\begin{tabular}{|c|c|c|c|c|c|c|c|}
\hline \multirow{2}{*}{$\begin{array}{l}\text { Time } \\
\text { (hours) }\end{array}$} & \multicolumn{6}{|c|}{ Dose (ppm) } & \multirow[t]{2}{*}{ Mean } \\
\hline & 500 & 250 & 125 & 62.50 & 31.25 & Control & \\
\hline 24 & $2.50 \pm 2.50$ & $0.00 \pm 0.00$ & $0.00 \pm 0.00$ & $0.00 \pm 0.00$ & $0.00 \pm 0.00$ & $0.00 \pm 0.00$ & $0.42 \pm 0.42 \mathrm{D}$ \\
\hline 48 & $12.50 \pm 2.50$ & $7.50 \pm 2.50$ & $5.00 \pm 2.89$ & $2.50 \pm 2.50$ & $0.00 \pm 0.00$ & $2.50 \pm 2.50$ & $5.00 \pm 1.20 \mathrm{C}$ \\
\hline 72 & $15.00 \pm 2.89$ & $12.50 \pm 2.50$ & $7.50 \pm 2.50$ & $5.00 \pm 2.89$ & $2.50 \pm 2.50$ & $2.50 \pm 2.50$ & $7.50 \pm 1.38 \mathrm{BC}$ \\
\hline 96 & $17.50 \pm 2.50$ & $12.50 \pm 2.50$ & $10.00 \pm 0.00$ & $10.00 \pm 0.00$ & $5.00 \pm 2.89$ & $2.50 \pm 2.50$ & $9.58 \pm 1.27 \mathrm{AB}$ \\
\hline 120 & $17.50 \pm 2.50$ & $15.00 \pm 2.89$ & $12.50 \pm 2.50$ & $10.00 \pm 0.00$ & $7.50 \pm 2.50$ & $5.00 \pm 2.89$ & $11.25 \pm 1.25 \mathrm{~A}$ \\
\hline 144 & $17.50 \pm 2.50$ & $15.00 \pm 2.89$ & $12.50 \pm 2.50$ & $10.00 \pm 0.00$ & $10.00 \pm 0.00$ & $5.00 \pm 2.89$ & $11.67 \pm 1.15 \mathrm{~A}$ \\
\hline Total & $13.75 \pm 1.45 \mathrm{~A}$ & $10.42 \pm 1.41 \mathrm{~B}$ & $7.92 \pm 1.20 \mathrm{BC}$ & $6.25 \pm 1.01 \mathrm{CD}$ & $4.17 \pm 1.03 \mathrm{DE}$ & $2.92 \pm 0.95 \mathrm{E}$ & \\
\hline
\end{tabular}

Means with the same letter in a row or in a column are not significantly different by Fisher's LSD (P $>0.05)$.

Table 6b. Rate of escape (\%) of $N$. barkeri $(\mathrm{n}=10)$ at different time intervals for imidacloprid (Mean $\pm \mathrm{SE})$.

\begin{tabular}{|c|c|c|c|c|c|c|c|}
\hline \multirow{2}{*}{$\begin{array}{l}\text { Time } \\
\text { (hours) }\end{array}$} & \multicolumn{6}{|c|}{ Dose (ppm) } & \multirow[t]{2}{*}{ Mean } \\
\hline & 500 & 250 & 125 & 62.50 & 31.25 & Control & \\
\hline 24 & $10.00 \pm 0.00$ & $10.00 \pm 0.00$ & $10.00 \pm 0.00$ & $7.50 \pm 2.50$ & $7.50 \pm 2.50$ & $7.50 \pm 2.50$ & $8.75 \pm 0.69 \mathrm{C}$ \\
\hline 48 & $12.50 \pm 2.50$ & $10.00 \pm 0.00$ & $10.00 \pm 0.00$ & $7.50 \pm 2.50$ & $7.50 \pm 2.50$ & $7.50 \pm 2.50$ & $9.17 \pm 0.83 \mathrm{BC}$ \\
\hline 72 & $12.50 \pm 2.50$ & $10.00 \pm 0.00$ & $10.00 \pm 0.00$ & $10.00 \pm 0.00$ & $7.50 \pm 2.50$ & $7.50 \pm 2.50$ & $9.58 \pm 0.73 \mathrm{ABC}$ \\
\hline 96 & $15.00 \pm 2.89$ & $12.50 \pm 2.50$ & $12.50 \pm 2.50$ & $10.00 \pm 0.00$ & $10.00 \pm 0.00$ & $7.50 \pm 2.50$ & $11.25 \pm 0.92 \mathrm{AB}$ \\
\hline 120 & $15.00 \pm 2.89$ & $12.50 \pm 2.50$ & $12.50 \pm 2.50$ & $10.00 \pm 0.00$ & $10.00 \pm 0.00$ & $7.50 \pm 2.50$ & $11.25 \pm 0.92 \mathrm{AB}$ \\
\hline 144 & $17.50 \pm 2.50$ & $12.50 \pm 2.50$ & $12.50 \pm 2.50$ & $10.00 \pm 0.00$ & $10.00 \pm 0.00$ & $7.50 \pm 2.50$ & $11.67 \pm 0.98 \mathrm{~A}$ \\
\hline Total & $13.75 \pm 1.01 \mathrm{~A}$ & $11.25 \pm 0.69 \mathrm{~B}$ & $11.25 \pm 0.69 \mathrm{~B}$ & $9.17 \pm 0.58 \mathrm{BC}$ & $8.75 \pm 0.69 \mathrm{C}$ & $7.50 \pm 0.90 \mathrm{C}$ & \\
\hline \multicolumn{8}{|c|}{ Means with the same letter in a row or in a column are not significantly different by Fisher's LSD (P>0.05). } \\
\hline \multicolumn{2}{|c|}{ Tested pesticides } & \multicolumn{2}{|c|}{$\begin{array}{c}\text { Observed mortality (\%) } \\
\text { after } 144 \text { hours }\end{array}$} & \multicolumn{2}{|c|}{$\begin{array}{c}\text { Categories of pesticides according } \\
\text { to IOBC (Jansen, 2010) }\end{array}$} & \multicolumn{2}{|c|}{$\begin{array}{c}\text { Categories of tested } \\
\text { pesticides }\end{array}$} \\
\hline \multicolumn{2}{|c|}{ buprofezin } & \multicolumn{2}{|c|}{17.5} & \multicolumn{2}{|c|}{$0-25 \%$ (Harmless) } & \multicolumn{2}{|c|}{ Harmless } \\
\hline \multicolumn{2}{|c|}{ imidacloprid } & \multicolumn{2}{|c|}{17.5} & \multicolumn{2}{|c|}{$0-25 \%$ (Harmless) } & \multicolumn{2}{|r|}{ Harmless } \\
\hline \multicolumn{2}{|c|}{ hexithiazox } & \multicolumn{2}{|c|}{35.0} & \multicolumn{2}{|c|}{$25-50 \%$ (Slightly harmful) } & \multicolumn{2}{|c|}{ Slightly harmful } \\
\hline \multicolumn{2}{|c|}{ spirotetramate } & \multicolumn{2}{|c|}{45.0} & \multicolumn{2}{|c|}{$25-50 \%$ (Slightly harmful) } & \multicolumn{2}{|c|}{ Slightly harmful } \\
\hline \multicolumn{2}{|c|}{ dimethoate } & \multicolumn{2}{|c|}{82.5} & \multicolumn{2}{|c|}{$>75 \%($ Harmful $)$} & \multicolumn{2}{|c|}{ Harmful } \\
\hline
\end{tabular}

Table 8. LC $\mathrm{LC}_{50}$ values of tested pesticides against $N$. barkeri after 144 hours.

\begin{tabular}{lcccccc}
\hline Pesticides & LC $_{\mathbf{5 0}}$ & SE & \multicolumn{2}{c}{ 95\% Fiducial CI } & Chi-square & P-Value \\
\cline { 4 - 6 } & & & Upper & Lower & & \\
\hline buprofezin & 7209.62 & 4944.55 & 2846.91 & 91452.50 & 3.231 & 0.357 \\
imidacloprid & 26526.66 & 60019.92 & 2335.84 & $1.8063 \mathrm{E}+17$ & 0.249 & 0.969 \\
spirotetramate & 682.31 & 109.15 & 520.78 & 1009.77 & 0.647 & 0.886 \\
"dimethoate & - & - & - & - & - & - \\
hexithiazox & 179.59 & 36.63 & 129.33 & 305.73 & 1.312 & 0.726 \\
\hline
\end{tabular}

CI: Confidence Interval, P: Probability, ${ }^{*}$ No Neoseiulus barkeri survived on arena after 120 hours (82.50\% dead; $17.50 \%$ escaped).

concentrations for escape $(\mathrm{F}=0.14, \mathrm{df}=25,108, \mathrm{P}=1.000)$ and mortality $(\mathrm{F}=1.25, \mathrm{df}=25,108, \mathrm{P}=0.2154)$ (Table $3 \mathrm{a}, \mathrm{b})$. Dimethoate was harmful for $N$. barkeri and complete mortality was observed at field relevant dose (1600 ppm) after 120 hours while minimum mortality $5 \%$ was observed at 100 ppm after 24 and 48 hours interval (Table 4a). Significant interaction of time and concentrations for escape of mites $(\mathrm{F}$ $=1.73, \mathrm{df}=25,108, \mathrm{P}=0.028)$ and mortality $(\mathrm{F}=17.17, \mathrm{df}=$ $25,108, \mathrm{P} \leq 0.000$ ) was observed (Table $4 \mathrm{a}, \mathrm{b}$ ).

Maximum mortality of $N$. barkeri (35\%) was reported at field relevant dose (125 ppm) of hexithiazox after 120 hours while minimum mortality (2.50\%) at 15.62 and $7.81 \mathrm{ppm}$ after 24 and 96 hours respectively (Table 5a). There was no interaction between time and concentrations for escape of mites $(\mathrm{F}=0.182, \mathrm{df}=25,108, \mathrm{P}=1.000)$ and mortality $(\mathrm{F}=$ 0.885, $\mathrm{df}=25,108, \mathrm{P}=0.624)$ (Table 5a, b).

Imidacloprid was harmless and caused maximum mortality $(17.5 \%)$ at field relevant dose (500 ppm) after 96 hours, while minimum mortality $(2.5 \%)$ at same concentration after 24 hours (Table 6a). There was no interaction between time and concentrations for escape $(\mathrm{F}=0.24, \mathrm{df}=25,108, \mathrm{P}=0.999)$ and mortality $(\mathrm{F}=0.85, \mathrm{df}=25,108 \mathrm{P}=0.668)($ Table $6 \mathrm{a}, \mathrm{b})$. 
Table 9. LT $_{50}$ values of tested pesticides against $N$. barkeri.

\begin{tabular}{|c|c|c|c|c|c|c|}
\hline \multirow[t]{2}{*}{ Pesticides } & \multirow[t]{2}{*}{$\mathbf{L T}_{50}$} & \multirow[t]{2}{*}{ SE } & \multicolumn{2}{|c|}{ 95\% Fiducial CI } & \multirow[t]{2}{*}{ Chi square } & \multirow[t]{2}{*}{ P Value } \\
\hline & & & Upper & Lower & & \\
\hline buprofezin & 411.35 & 111.03 & 275.13 & 901.38 & 6.132 & 0.804 \\
\hline imidacloprid & 332.38 & 70.63 & 239.63 & 594.80 & 9.810 & 0.457 \\
\hline spirotetramate & 113.30 & 12.10 & 94.07 & 146.33 & 1.713 & 0.998 \\
\hline dimethoate & 27.76 & 1.80 & 24.05 & 31.12 & 14.775 & 0.039 \\
\hline hexithiazox & 203.59 & 34.09 & 156.10 & 317.21 & 1.078 & 1.000 \\
\hline
\end{tabular}

Table 10. Biofilm inhibition and hemolytic activities of tested pesticides.

\begin{tabular}{lcc}
\hline Name of Pesticide & Staphylococcus aureus $\mathbf{( \% )}$ & Hemolytic activity (\%) \\
\hline imidacloprid & 26 & 78.56 \\
buprofezin & 43 & 94.57 \\
spirotetramate & 26 & 87.05 \\
hexithiazox & 36 & 88.20 \\
dimethoate & 23 & 97.76 \\
Rimpacin & 87.43 & - \\
PBS & - & 0.086 \\
Triton-x-100 & - & 98.85 \\
\hline
\end{tabular}

Values (mean \pm SD) are average of three samples of each formulated pesticides, analyzed individually in triplicate $(\mathrm{n}=1 \mathrm{x} 3 \mathrm{x} 3),(\mathrm{P}<$ 0.05); PBS: Phosphate Buffer Saline

According to IOBC category of tested pesticides against beneficial arthropods (Jansen, 2010) imidacloprid and buprofezin were harmless while spirotetramate and hexithiazox were slightly harmful and dimethoate was harmful for $N$. barkeri (Table 7). After 144 hours LC $_{50}$ values were 26526, 7209, 682 and 179 for imidacloprid, buprofezin, spirotetramate and hexithiazox respectively. The complete mortality due to dimethoate after 120 hours declared it highly toxic for $N$. barkeri and this pesticide cannot be recommended in IPM programs along with this predator (Table 8). However, $\mathrm{LC}_{50}$ did not indicate the clear mechanism of mortality due to difference of field relevant dose/ppm of all tested pesticides. So, $\mathrm{LT}_{50}$ were also calculated, which varied significantly: dimethoate $(27.76)<$ spirotetramate $(113.30)<$ hexithiazox (203.59) < imidacloprid (332.38) < buprofezin (411.35) (Table 9). For biofilm inhibition rimpacin was used as positive control. Staphylococcus aureus biofilm inhibition effect was lowest for dimethoate and highest for buprofezin as, 23, 26, 26, 36 and 43 percent for dimethoate, spirotetramate, imidacloprid, hexithiazox and buprofezin, respectively. The results indicated that gram-positive bacterium $S$. aureus biofilm inhibition was high due to the absence of slime layer. Erythrocytes lysis for each pesticide sample showed the least cytotoxicity $(78.56 \%)$ due to imidacloprid, while the highest cytotoxicity $(97.76 \%)$ due to dimethoate in contrast to positive control (Triton-x-100) (98.85\%) (Table 10). Biofilm inhibition microscopy of tested pesticides showed highest biofilm inhibition due to buprofezin as treatment when tested against $S$. aureus positive (rimpacin), negative (growth) and sample (growth) (Fig. 1).

\section{MICROSCOPY}

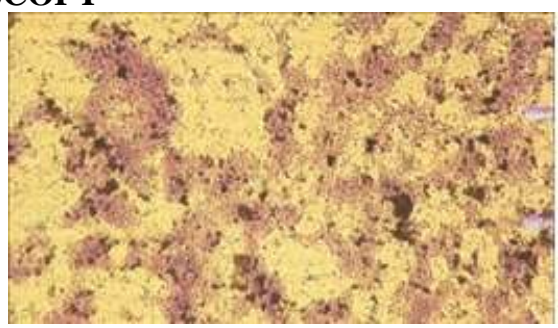

Treatment (Buprofezin)

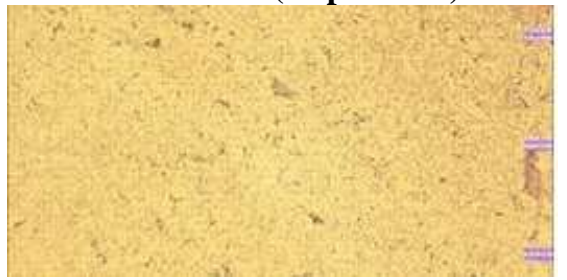

Negative control (Simple nutrient agar microbes growth)

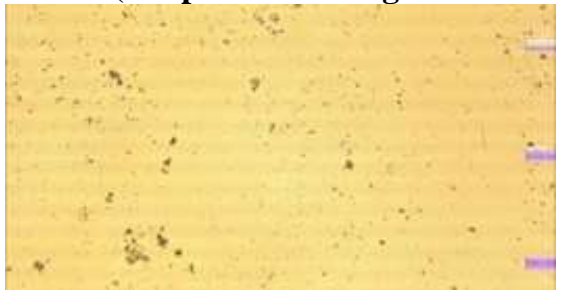

Positive control (Rimpacin)

Figure 1. Phase contrast microscopic view of inhibition of Staphylococcus aureus biofilm by pesticides nanoparticles at 100x. Positive (Triton-x-100), biofilm treated $S$. aureus growth by pesticides nanoparticles dissolved. 


\section{DISCUSSION}

Mortality and repellent effects due to tested pesticides varied significantly for N. barkeri (Acari: Phytoseiidae). Buprofezin in the present study proved to be least harmful pesticide at field relevant dose. These outcomes agree with the findings of Kongchuensin and Takafuji (2006) who observed least mortality (4.9\%) of Neoseiulus longispinosis due to buprofezin after 48 hours and declared harmless pesticide. The present results are also in agreement with IOBC/wprs recommendations (Boller et al., 2006) that buprofezin at dose rate $250 \mathrm{~g} / \mathrm{L}$ was harmless against Typhlodromus pyri and Phytoseiulus persimilis. Spirotetramate had slightly harmful affect against $N$. barkeri. Present findings are in consistence with outcomes of Beers and Schmidt (2014) who tested spirotetramate against Galendromus occidentalis at different dose rates i.e., 328(2x), 164(x), 16(0.1x) and $0 \mathrm{mg}$ active ingredients per liter, and found 52, 20.83, 48 and $12.5 \%$ mortality after 48 hours, respectively. Beers and Schmidt (2014) declared spirotetramate as slightly harmful against predatory mites like the results presented here. The present study confirmed that dimethoate was harmful. Similarly, Bostanian and Akalach (2006) tested dimethoate at dose rate 0.190 grams active ingredients per liter against $P$. persimilis and Amblyseius fallacis and found $100 \%$ and $94 \%$ mortality after 168 hours, respectively. Their outcomes are in consistence to present findings. Alzoubi and Cobanoglu (2008) tested dimethoate at dose rate $450 \mathrm{ppm}$ against Amblyseius californicus and P. persimilis and observed $\mathrm{LC}_{50}$ 33.76 and 5.54 respectively. Their findings agree with the present results that dimethoate had lethal effects against predatory mites. Dimethoate was also found harmful for $T$. pyri in field conditions and for $P$. persimilis in laboratory conditions according to IOBC/wprs. These results indicated its higher toxicity for predatory mites and dimethoate is not recommended in IPM module.

Hexithiazox at dose rate $50 \mathrm{ppm}$ tested against $A$. californicus and $P$. persimilis by Alzoubi and Cobanoglu (2008), who observed $\mathrm{LC}_{50}, 280.98,50.61$ and 184.48, 28.92 after 24 and 72 hours respectively. Their conclusion is in consistence to present findings that hexithizox is suitable for IPM programs due to slightly harmful effects against tested predatory mite. Sanatgar et al. (2011) also observed the least toxicity of hexithiazox for different generations of $P$. persimilis in consistence to present findings. Fiedler and Sosnowska (2014) tested hexithiazox at $0.02 \%$ dose rate against Amblyseius swiriskii, Amblyseius andersoni and P. persimilis and found 8, 12 and 20\% mortality after 168 hours, respectively. Hexithiazox tested by IOBC/ wprs at dose rate 200 grams per liter against $T$. pyri in field conditions and $P$. persimilis in laboratory conditions was found slightly harmful. Present findings revealed that imidacloprid was least harmful, which agrees with the outcomes of Castagnoli et al. (2005) who tested imidacloprid at $13.3 \mathrm{~g}$ a.i./hl against
Neoseiulus californicus and found $1.27 \%$ mortality after 72 hours. Their results are different from present findings due to difference of dose and tested species. Imidacloprid was tested at $50 \mathrm{ppm}$ dose rate against Neoseiulus longispinosis by Kongchuensin and Takafuji (2006), who reported 20.2\% mortality in 48 hours. Their findings are somewhat in agreement with present results because imidacloprid falls in harmless category of IOBC in both studies. Beers and Schmidt (2014) tested imidacloprid against G. occidentalis at dose rate of $120 \mathrm{mg}$ active ingredients per liter (x) and found 80,68 and $12 \%$ mortality at $2 \mathrm{x}, 1 \mathrm{x}, 0.1 \mathrm{x}$ dose rates along with $8 \%$ mortality at control after 48 hours. Present results are not in agreement with their findings due to difference of species and conditions, but somewhat similar because the author also found 5\% mortality in control conditions. Villanueva and Walgenbach (2005) tested imidacloprid at $60 \mathrm{ppm}$ dose rate against Neoseiulus fallacis and found $1.3 \%$ mortality and $8.8 \%$ escape after 24 hours, while $20 \%$ mortality and escape reported after 96 hours. Their results showed the similar trend of mortality and missing as present findings. Fiedler and Sosnowska (2014) tested imidacloprid at $0.075 \%$ dose rate against A. swiriskii, Amblyseius andersoni and P. persimilis and found 92, 98 and 100\% mortality after 168 hours, respectively. Moreover, according to IOBC/wprs, imidacloprid was found harmless against $T$. pyri and harmful for $P$. persimilis. Present results are also in disagreement with IOBC due to difference of species used in both cases.

Present study is the first to screen out reduced risk pesticides against $N$. barkeri under laboratory conditions. Buprofezin and imidacloprid caused the lowest mortality, while dimethoate resulted highest mortality. However, further investigations are still needed to study sub-lethal effects of these pesticides on subsequent generations of $N$. barkeri. Present study declared hexithiazox and spirotetramate to be slightly harmful against $N$. barkeri in laboratory conditions. Resultantly, buprofezin and imidacloprid can be recommended for use at their field relevant dose rates, hexithiazox and spirotetramate can be used at lower dose rates, while dimethoate is not recommended in IPM module.

Conclusion: Hexithiazox and spirotetramate declared slightly harmful against Neoseiulus barkeri under laboratory conditions. Buprofezin and imidacloprid can be recommended for use at their field relevant dose rates, hexithiazox and spirotetramate can be used at lower dose rates, while dimethoate is not recommended in IPM module. Biochemical analysis also revealed that imidacloprid and buprofezin were the safest pesticides while dimethoate declared as highly toxic for non-target organisms.

Acknowledgement: The authors acknowledge Higher Education Commission (HEC) Pakistan for financial support in the form of Ph.D. indigenous scholarship scheme for timely completion of this piece of research work. 


\section{REFERENCES}

Abang, A.F., C.M. Kouame, M. Abang, R. Hanna and A.K. Fotso. 2013. Vegetable grower's perception of pesticide use practices, cost and health effects in the tropical region of Cameroon. Int. J. Agron. Plant Prod. 4:873-883.

Ahmad, S., Noor-ul-Islam, A. Mahmood, F. Ashraf, K. Hayat and M. Hanif. 2010. Screening of cotton germplasm against cotton leaf curl virus. Pak. J. Bot. 42:3327-3342.

Ajinath, S.D., R. Prasanna, L. Nain and A.K. Saxena. 2013. Optimization and evaluation of microbe fortified composts as biocontrol agents against phytopathogenic fungi. J.M.B.F.S. 2:2272-2276.

Alavanja, M.C.R. and M.R. Bonner. 2012. Occupational pesticide exposures and cancer risk: a review. J. Toxicol. Environ. Health B. Crit. Rev. 15:238-63.

Alzoubi, S. and S. Cobanoglu. 2008. Toxicity of some pesticides against Tetranychus urticae and its predatory mites under laboratory conditions. Am-Euras. J. Agric. Environ. Sci. 3:30-37.

Attia, S., K.G. Lebdi, S. Heuskin, G. Lognay and T. Hance. 2015. An analysis of potential resistance of the phytophagous mite, Tetranychus urticae Koch (Acari: Tetranychidae) to four botanical pesticides. Biotechnol. Agron. Soc. 19:232-238.

Bakker, F.M. and M.W. Sabelis. 1989. How larvae of Thrips tabaci reduce the attack success of phytoseiid predators. Entomol. Exp. Appl. 50:47-51.

Beers, E.H. and R.A. Schmidt. 2014. Impacts of orchard pesticides on Galendromus occidentalis: Lethal and sublethal effects. Crop Prot. 56:16-24.

Bernard, M.B., P. Cole, A. Kobelt, P.A. Horne, J. Altmann, S.D. Wratten and A.L. Yen. 2010. Reducing the impact of pesticides on biological control in Australian vineyards: pesticide mortality and fecundity effects on an indicator species, the predatory mite Euseius victoriensis (Acari: Phytoseiidae). J. Econ. Entomol. 103:2061-2071.

Boldt, T.S. and C.S. Jacobsen. 1998. Different toxic effects of the sulphonylurea herbicides metsulfuron methyl, chlorsulfuron and thifensulfuron methyl on fluorescent Pseudomonads isolated from an agricultural soil. FEMS Microbiol. Lett. 161:29-35.

Boller, E.F., H. Vogt, P. Ternes and C. Malavolta. 2006. Working document on selectivity of pesticides (2005). Internal newsletter issued by the publication commission for the IOBC/wrps council and executive committee issue, (40).

Bonde, J. 1989. Biological studies including population growth parameters of the predatory mite Neoseiulus barkeri (Acarina: Phytoseiidae) at $25^{\circ} \mathrm{C}$ in the laboratory. Entomophaga. 34:275-287.

Bostanian, N.J. and M. Akalach. 2006. The effect of indoxacarb and five other insecticides on Phytoseiulus persimilis (Acari: Phytoseiidae), Amblyseius fallacis
(Acari: Phytoseiidae) and nymphs of Orius insidiosus (Hemiptera: Anthocoridae). Pest Manage. Sci. 62:334339.

Bostanian, N.J., H.M.A. Thistlewood, J.M. Hardman, M.C. Laurin and G. Racette. 2009. Effect of seven new orchard pesticides on Galendromus occidentalis in laboratory studies. Pest Manage. Sci. 65:635-639.

Castagnoli, M., M. Liguori, S. Simoni and C. Duso. 2005. Toxicity of some insecticides to Tetranychus urticae, Neoseiulus californicus and Tydeus californicus. Bio. Control 50:611-622.

Chant, D.A. and J.A. McMurtry. 2007. Illustrated keys and diagnoses for the genera and subgenera of the Phytoseiidae of the world (Acari: Mesostigmata). Indira Publishing House, West Bllomfield, Michigan, USA. p.220.

Fan, Y.Q. and F.L. Petit. 1994. Biological control of broad mite, Polyphagotarsonemus latus (Banks), by Neoseiulus barkeri Hugues on pepper. Biol. Control. 4:390-395.

Fiedler, Z. and D. Sosnowska. 2014. Side effects of fungicides and insecticides on predatory mites, in laboratory conditions. J. Plant Prot. Res. 54:349-353.

Giglio, A., P.G. Giulianini, T. Zetto and F. Talarico. 2011. Effects of the pesticide dimethoate on a non-target generalist carabid, Pterostichus melas italicus (Dejean, 1828) (Coleoptera: Carabidae). Ital. J. Zool. 78:471-477.

Hansen, L.S. 1988. Control of Thrips tabaci (Thysanoptra: Thripidae) on glasshouse cucumber using large introductions of predatory mites Amblyseius barkeri (Acarina: Phytoseiidae). Entomophaga. 33:33-42.

Hosny, A.H., A.A. Ismail and A.Y. Keratum. 2009. Integrated mites management II-Determination of sub lethal doses of some compounds against the two spotted spider mite Tetranychus urticae and their effects on its biological aspects with respect to predators Amblyseius fallacies and Phtodeiulus persimilis. J. Agric. Res. Kafrel Sheikh Univ. 35:1096-1113.

Ibekwe, A.M., S.K. Papiernik, J. Gan, S.R. Yates, C.H. Yang and D.E. Crowley. 2001. Impact of fungicides on soil microbial communities. Appl. Environ. Microbiol. 67:3245-3257.

Jafari, S., N. Abassi and F. Bahirae. 2013. Demographic parameters of Neoseiulus barkeri (Acari: Phytoseiidae) fed on Thrips tabaci (Thysanoptera: Thripidae). P.J.A. 2:287-296.

Jansen, J.P. 2010. Beneficial arthropods and pesticides: building selectivity list for IPM. IOBC/WPRS Bull. 55:23-47.

Kongchuensin, M. and A. Takafuji. 2006. Effects of some pesticides on the predatory mite, Neoseiulus longispinosus (Evans) (Gamasina: Phytoseiidae). J. Acarol. Soc. Jpn. 15:17-27.

Kumar, R., S. Kranthi, M. Nitharwal, S.L. Jat and D. Monga. 2012. Influence of pesticides and application methods on 
pest and predatory arthropods associated with cotton. Phytoparasitica 40:417-424.

Lechenet, M., V. Bretagnolle, C. Bockstaller, F. Boissinot, MS. Petit, S. Petit and N.M. Munier-Jolain. 2014. Reconsiling pesticide reduction with economic and environmental sustainability in arable farming. PLoS One 9:1-10.

Lefebvre, M., N.J. Bostanian, Y. Mauffette, G. Racette, H.A. Thistlewood and J.M. Hardman. 2012. Laboratory-based toxicological assessments of new insecticides on mortality and fecundity of Neoseiulus fallacis (Acari: Phytoseiidae). J. Econ. Entomol. 105: 866-871.

Mallik, B., C.C. Gowda, N. Srinivasa and K. Rajashekarappa. 2010. Phytoseiid mites as biocontrol agents Indian Scenario. International Symposium-cum-Workshop in Acarology, BCKV, Kalyani, p.117.

Malik, K.A. 2014. Biotechnology in Pakistan status and prospectus. Pakistan Academy of Sciences, G-5/2 Islamabad. p.60.

Mamoon-ur-Rashid, M., M.K. Khattak, K. Abdullah and S. Hussain. 2011. Toxic and residual activities of selected insecticides and neem oil against cotton mealy bug, Phenacoccus solenopsis Tinsley (Sternorrhyncha: Pseudococcidae) under laboratory and field conditions. Pak. Entomol. 33:151-155.

McMurtry, J.A., G.J.D. Moraes and N.F. Sourassou. 2013. Revision of the lifestyles of phytoseiid mites (Acari: Phytoseiidae) and implications for biological control strategies. Syst. Appl. Acarol. 18:297-320.

Messelink, G.J. and R. van Holstein-Saj. 2007. Biological control of the bulb scale mite Steneotrasonemus laticeps (Acari: Tarsonemidae) with Neoseiulus barkeri (Acari: Phytoseiidae) in amaryllis. IOBC/WPRS Bulletin 30:8185.

Messelink, G.J., M.W. Sabelis and A. Janssen. 2012. Generalist predators, food web complexities and biological pest control in greenhouse crops, integrated pest management and pest control-current and future tactics, Dr. Sonia Soloneski (Ed.), InTech DOI: 10.5772/30835.

Negm, M.W., F.J. Alatawi and Y.N. Aldryhim. 2014. Biology, predation, and life table of Cydnoseius negevi and Neoseiulus barkeri (Acari: Phytoseiidae) on the oldworld date mite, Oligonychus afrasiaticus (Acari: Tetranychidae). J. Insect Sci. 14:177.

Rafiq, M., A. Ghaffar and M. Arshad. 2008. Population dynamics of whitefly (Bemisia tabaci) on cultivated crop hosts and their role in regulating its carry-over to cotton. Int. J. Agric. Biol. 10:577-80.

Sanatgar, E., R.V. Shoushtari, A.A. Zamani, M. Arbabi and E.S. Nejadian. 2011. Effect of Frequent Application of Hexythiazox on Predatory Mite Phytoseiulus persimilis Athias - Henriot (Acari: Phytoseiidae). Acad. J. Entomol. 4:94-101.

Son, H., S. Kim, J. Hang, Y. Chang, S. Park, S. Lee, D. Jaco bs and D. Lee. 2010. Strong associations between low dose organochlorine pesticides and type 2 diabetes in Korea. Environ. Int. 36:410-414.

Szabo, A., B. Penzes, P. Sipos, T. Hegyi, Z. Hajdu and V. Marko. 2014. Pest management systems affect composition but not abundance of phytoseiid mites (Acari: Phytoseiidae) in apple orchards. Exp. Appl. Acarol. 62:525-537.

Tirello, P., A. Pozzebon and C. Duso. 2013. The effect of insecticides on the non-target predatory mite Kampimodromus aberrans: laboratory studies. Chemosphere 93:1139-44.

Villanueva, R.T. and J.F. Walgenbach. 2005. Development, oviposition, and mortality of Neoseiulus fallacis (Acari: Phytoseiidae) in response to reduced-risk insecticides. J. Econ. Entomol. 98:2114-2120.

Xia, B., Z. Zou, P. Li and P. Lin. 2012. Effect of temperature on development and reproduction of Neoseiulus barkeri (Acari: Phytoseiidae) fed on Aleuroglyphus ovatus. Exp. Appl. Acarol. 56: 33-41. 